\title{
Outer automorphisms of free Burnside groups
}

\author{
Rémi Coulon
}

\begin{abstract}
In this paper, we study some properties of the outer automorphism group of free Burnside groups of large odd exponent. In particular, we prove that it contains free and free abelian subgroups.
\end{abstract}

Mathematics Subject Classification (2010). 20F65, 20F50, 20E36, 20 F06.

Keywords. Geometric group theory, periodic groups, automorphism groups, hyperbolic geometry, small cancellation theory.

\section{Introduction}

The free Burnside group of rank $r$ and exponent $n$, denoted by $\mathbf{B}_{r}(n)$, is the quotient of the free group $\mathbf{F}_{r}$ by the subgroup $\mathbf{F}_{r}^{n}$ generated by the $n$-th powers of all its elements. In 1902, W. Burnside asked whether $\mathbf{B}_{r}(n)$ had to be finite of not (see [9]). For a long time, one only knew that for some small exponents $\mathbf{B}_{r}(n)$ was finite (for $n=2$ see [9], $n=3$ [9] and [22], $n=4$ [31] and $n=6$ [21]). In 1968, P. S. Novikov and S. I. Adian achieved a breakthrough (see [25], [26] and [27]). Using the small cancellation theory developed by V. A. Tartakovskiu [33] and M. Greendlinger [17], [18], [19], they proved that for large odd exponents, $\mathbf{B}_{r}(n)$ is infinite. Thanks to a diagrammatic formulation of small cancellation, A. Y. Ol'shanskiur simplified the proof of P. S. Novikov and S. I. Adian [28]. Recently, T. Delzant and M. Gromov gave a more geometrical proof of the same theorem [15]. These results not only provide examples of infinite Burnside groups, they also help to study many of their properties (solution for the word-problem, description of finite subgroups,...). Other information about the history of the Burnside problems can be found in [20].

The next step to understand Burnside groups is to study their automorphisms. In this paper, we are interested in the following questions. What kind of outer automorphisms of $\mathbf{B}_{r}(n)$ have infinite order? Does Out $\left(\mathbf{B}_{r}(n)\right)$ contain relevant subgroups like free groups or free abelian groups? To that end, we focus on the canonical map Out $\left(\mathbf{F}_{r}\right) \rightarrow$ Out $\left(\mathbf{B}_{r}(n)\right)$.

Using the work of P. S. Novikov and S. I. Adian, E. A. Cherepanov proved that the automorphism $\varphi$ of $\mathbf{F}_{2}=\mathbf{F}(a, b)$, defined by $\varphi(a)=a b$ and $\varphi(b)=a$, induces 
an outer automorphism of infinite order of $\mathbf{B}_{r}(n)$ (see [10] and Proposition 1.1). Our first theorem provides a large class of automorphisms of the free group having the same property.

Theorem 1 (see Theorem 1.3). Let $\varphi$ be an automorphism of $\mathbf{F}_{r}$. Assume that $\varphi$ is hyperbolic, i.e. the semi-direct product $\mathbf{F}_{r} \rtimes_{\varphi} \mathbb{Z}$ defined by $\varphi$ is a hyperbolic group. There exists an integer $n_{0}$ such that for all odd integers $n$ larger than $n_{0}, \varphi$ induces an outer automorphism of infinite order of $\mathbf{B}_{r}(n)$.

All proofs dealing with free Burnside groups have the same weakness: they involve a presentation of $\mathbf{B}_{r}(n)$ which is not stable under automorphisms. Our work tries to regain a little symmetry: we build a sequence of groups $\left(H_{k}\right)$ such that $\varphi$ induces an automorphism of $H_{k}$ for all $k$ and $\lim H_{k}=\mathbf{B}_{r}(n)$. To that end, we start with $H_{0}=\mathbf{F}_{r}$ and, at each step, we construct $H_{k+1}$ as a small cancellation quotient of $H_{k}$. Some difficulties appear during this process. Assume that $\rho$ is one of the relations defining the first quotient $\mathbf{F}_{r}=H_{0} \rightarrow H_{1}$. Since we want $\varphi$ to induce an automorphism of $H_{1}$, the elements $\varphi^{m}(\rho)$ for all $m \in \mathbb{N}$ have to belong to the set of relations. However the small cancellation theory only deals with relations having more or less the same length. In our case, the relations $\varphi^{m}(\rho)$ may have very different lengths. To avoid this problem, we encode the information concerning the automorphism in a larger group: $\mathbf{F}_{r} \rtimes_{\varphi} \mathbb{Z}$. Thus the elements $\varphi^{m}(\rho)$ become conjugates of $\rho$ and do not need to be added to the set of relations. We shall now use the fact that the group $\mathbf{F}_{r} \rtimes \mathbb{Z}$ is hyperbolic. In 1991, A.Y. Ol'shanskiı provided a generalisation of the Novikov-Adian theorem (see [29]). Given a torsion-free, hyperbolic group $G$, he proved that for large odd exponent $n$ the quotient $G / G^{n}$ is infinite. This result was recovered by T. Delzant and M. Gromov in [15]. We would like to apply the same techniques to $G=\mathbf{F}_{r} \rtimes \mathbb{Z}$. However we must take care not to kill all $n$-th powers of $G$. Indeed, if we did so, the automorphism obtained at the end of the construction would have finite order dividing $n$. That is why we propose an extension of the Delzant-Gromov construction where the relations are chosen in a normal subgroup of $\mathbf{F}_{r} \rtimes \mathbb{Z}$. This construction works in a more general situation. It leads to our main theorem:

Main theorem. Let $1 \rightarrow H \rightarrow G \rightarrow F \rightarrow 1$ be a short exact sequence of groups. Assume that $H$ is non-trivial, finitely generated, $G$ is hyperbolic, non-elementary, torsion-free and $F$ is torsion-free. There exists an integer $n_{0}$ such that for all odd integers $n$ larger than $n_{0}$, the canonical map $F \rightarrow$ Out $(H)$ induces an injective homomorphism $F \hookrightarrow$ Out $\left(H / H^{n}\right)$.

Theorem 1 is obtained by applying the main theorem to the short exact sequence $1 \rightarrow \mathbf{F}_{r} \rightarrow \mathbf{F}_{r} \rtimes \mathbb{Z} \rightarrow \mathbb{Z} \rightarrow 1$. The work of M. Bestvina, M. Feighn and M. Handel (see [5]) provides examples of hyperbolic extensions of free groups by free groups. Using this result, we obtain our second theorem. 
Theorem 2 (see Theorem 1.8). Let $r \geqslant 3$. There exists an integer $n_{0}$ such that for all odd integers $n$ larger than $n_{0}$, the group Out $\left(\mathbf{B}_{r}(n)\right)$ contains a subgroup which is isomorphic to $\mathbf{F}_{2}$.

The strategy to embed abelian subgroups in Out $\left(\mathbf{B}_{r}(n)\right)$ is a little different. We do not apply the main theorem to an appropriate hyperbolic extension of the free group. We construct a family of automorphisms of $\mathbf{F}_{r}$ which already commute in Aut $\left(\mathbf{F}_{r}\right)$ and check "by hand" that they do not satisfy any other relation in Out $\left(\mathbf{B}_{r}(n)\right)$. This yields the following result.

Theorem 3 (see Theorem 1.10). Let $r \geqslant 1$. There exists an integer $n_{0}$ such that for all odd integers $n$ larger than $n_{0}$, the groups Out $\left(\mathbf{B}_{2 r}(n)\right)$ and $\operatorname{Out}\left(\mathbf{B}_{2 r+1}(n)\right)$ contain a subgroup which is isomorphic to $\mathbb{Z}^{r}$.

Hyperbolic automorphisms induce automorphisms of infinite order of free Burnside groups of large exponent. But they are not the only ones. For instance, the automorphism $\varphi$, studied by E. A. Cherepanov and characterized by $\varphi(a)=a b$ and $\varphi(b)=a$ is not hyperbolic. Indeed, $\varphi^{2}$ fixes the commutator $\left[a^{-1}, b^{-1}\right]$. The semidirect product $\mathbf{F}_{r} \rtimes_{\varphi} \mathbb{Z}$ contains therefore a subgroup which is isomorphic to $\mathbb{Z}^{2}$. We wonder if there exists a criterion to decide whether an automorphism of $\mathbf{F}_{r}$ induces an outer automorphism of infinite order of $\mathbf{B}_{r}(n)$ for some large exponent or not. In particular, is there a link between this property and the growth of the automorphism? Section 1.2 gives a partial answer. We prove that a polynomially growing automorphism always induces an automorphism of finite order of $\mathbf{B}_{r}(n)$.

Outline of the paper. In Section 1 we explain the consequences of the main theorem. In particular, we provide examples of automorphisms of infinite order of $\mathbf{B}_{r}(n)$. We also construct free and free abelian subgroups of Out $\left(\mathbf{B}_{r}(n)\right)$. Section 2 deals with the proof of the main theorem. To begin with, we recall the geometrical point of view on the small cancellation theory developed by T. Delzant and M. Gromov. We also improve some results of [15] which are necessary to control the small cancellation parameters in our situation. Then, we prove an induction lemma (Lemma 2.16) which is the fundamental step of the induction process used in Section 3 to prove the main theorem.

Acknowledgement. I am grateful to Thomas Delzant for his invaluable help and advice during this work. I would like to thank Gilbert Levitt for related discussions, in particular, concerning the growth of automorphisms. Many thanks also go to Étienne Ghys who points out many questions to me, like the embedding of free abelian subgroups. I thank also the referee for many useful comments and corrections. 


\section{Automorphisms of Burnside groups}

Remark. In this paper, we are interested in outer automorphisms of free Burnside groups. One question still unanswered is the following: given an automorphism of the free group $\mathbf{F}_{r}$, does it induce an automorphism of infinite order of $\mathbf{B}_{r}(n)$ ? Note that every element of $\mathbf{B}_{r}(n)$ has finite order. In particular, every inner automorphism of $\mathbf{B}_{r}(n)$ has finite order. It follows that an element of Aut $\left(\mathbf{B}_{r}(n)\right)$ has finite order if and only if so has its image in Out $\left(\mathbf{B}_{r}(n)\right)$.

1.1. Examples of automorphisms of infinite order. Using the work of P. S. Novikov and S. I. Adian (see [25], [26] and [27]), we exhibit a first example of outer automorphism of infinite order of $\mathbf{B}_{r}(n)$. This example was already studied by E. A. Cherepanov in [10].

Proposition 1.1 (see [10], Theorem 1). Let $\{a, b\}$ be a generating set of the free group $\mathbf{F}_{2}$. Let $\varphi$ be the automorphism of $\mathbf{F}_{2}$ defined by $\varphi(a)=a b$ and $\varphi(b)=a$. There exists an integer $n_{0}$ such that for all odd integers $n$ larger than $n_{0}, \varphi$ induces an automorphism of infinite order of $\mathbf{B}_{2}(n)$.

Proof. We consider the sequence of iterated images of $a$ by $\varphi$.

$$
\begin{aligned}
\varphi^{0}(a) & =a, & \varphi^{4}(a) & =\text { abaababa }, \\
\varphi^{1}(a) & =a b, & \varphi^{5}(a) & =\text { abaababaabaab }, \\
\varphi^{2}(a) & =a b a, & \varphi^{6}(a) & =\text { abaababaabaababaababa }, \\
\varphi^{3}(a) & =a b a a b, & \ldots &
\end{aligned}
$$

This sequence converges to a right infinite positive word

$$
\varphi^{\infty}(a)=\text { abaababaabaababaababa } \ldots
$$

which has the following property. For every word $u$ in $\{a, b\}, u^{4}$ is not a subword of $\varphi^{\infty}(a)$ (see [24]). Let $n$ be an odd integer larger than 10000 . In order to prove that the free Burnside group of large exponent is infinite, P. S. Novikov and S .I. Adian use the following fact: if $m$ is a non-trivial reduced word in $\{a, b\}$ which does not contain a subword that equals a fourth power, then $m$ defines a non-trivial element of $\mathbf{B}_{2}(n)$ (see [1], IV. 2.16, or Statement 1 in [2]). In particular, $\left(\varphi^{p}(a)\right)$ induces a sequence of pairwise distinct elements of $\mathbf{B}_{2}(n)$. It follows that $\varphi$ induces an automorphism of infinite order of $\mathbf{B}_{2}(n)$.

We now wish to investigate a large class of automorphisms of free groups: the hyperbolic ones. We prove that they induce automorphisms of infinite order of free Burnside groups. 
Definition 1.2. Let $G$ be a hyperbolic group. An automorphism $\varphi$ of $G$ is hyperbolic if the semi-direct product $G \rtimes_{\varphi} \mathbb{Z}$ defined by $\varphi$ is hyperbolic.

Example. Let $\Sigma$ be the fundamental group of a compact surface $S$ of genus larger than 2. Thanks to Thurston's hyperbolisation Theorem, every pseudo-Anosov homeomorphism of $S$ induces a hyperbolic automorphism of $\Sigma$ (see [30]).

There exist many characterizations of hyperbolic automorphisms. Let us endow $G$ with the word metric | . | relative to a generating set. M. Bestvina and M. Feighn proved in [3] that an automorphism $\varphi$ of $G$ is hyperbolic if and only if there exist $\lambda>1$ and $m \in \mathbb{N}$ such that for all $g \in G$

$$
\lambda|g| \leqslant \max \left\{\left|\varphi^{m}(g)\right|,\left|\varphi^{-m}(g)\right|\right\} .
$$

On the other hand, an automorphism of a free group is hyperbolic, if and only if it has no non-trivial periodic conjugacy classes (see [5] and [8]). Note that the automorphism $\varphi$ studied in Proposition 1.1 is not hyperbolic: $\varphi^{2}$ fixes the commutator $\left[a^{-1} ; b^{-1}\right]$. More generally, Aut $\left(\mathbf{F}_{2}\right)$ does not contain hyperbolic elements. Every automorphism $\varphi$ of $\mathbf{F}_{2}$ is indeed induced by a homeomorphism of the punctured torus. Therefore $\varphi^{2}$ has to fix the conjugacy class of $\mathbf{F}_{2}$ corresponding to the boundary of the torus.

Theorem 1.3. Let $r \geqslant 3$. Let $\varphi$ be a hyperbolic automorphism of $\mathbf{F}_{r}$. There exists an integer $n_{0}$ such that for all odd integers $n$ larger than $n_{0}, \varphi$ induces an outer automorphism of infinite order of $\mathbf{B}_{r}(n)$.

Proof. By definition, the group $\mathbf{F}_{r} \rtimes_{\varphi} \mathbb{Z}$ is hyperbolic. It follows that the short exact sequence $1 \rightarrow \mathbf{F}_{r} \rightarrow \mathbf{F}_{r} \rtimes_{\varphi} \mathbb{Z} \rightarrow \mathbb{Z} \rightarrow 1$ satisfies the assumptions of the main theorem (see page 790). Thus there exists an integer $n_{0}$ such that for all odd integers $n$ larger than $n_{0}$, the map $\mathbb{Z} \rightarrow$ Out $\left(\mathbf{F}_{r}\right)$ induces an injective homomorphism $\mathbb{Z} \hookrightarrow$ Out $\left(\mathbf{B}_{r}(n)\right)$. However, the morphism $\mathbb{Z} \rightarrow$ Out $\left(\mathbf{F}_{r}\right)$ is by construction the one that maps $m$ to the outer automorphism induced by $\varphi^{m}$. Consequently, $\varphi$ induces an outer automorphism of infinite order of $\mathbf{B}_{r}(n)$.

1.2. Polynomially growing automorphisms of free groups. We provide now examples of automorphisms of infinite order of $\mathbf{F}_{r}$ which induce automorphisms of finite order of $\mathbf{B}_{r}(n)$. Given a conjugacy class $x$ of $\mathbf{F}_{r}$, we denote by $[x]$ the length of any cyclically reduced word representing $x$. Let $\Phi$ be an outer automorphism of $\mathbf{F}_{r}$. We look at the action of $\Phi$ on the conjugacy classes of $\mathbf{F}_{r}$.

Definition 1.4. The automorphism $\Phi$ grows polynomially if for every conjugacy class $x$ of $\mathbf{F}_{r}$, the sequence ([$\left.\left.\Phi^{p}(x)\right]\right)$ grows polynomially. 
Proposition 1.5 (see [23]). Let $\Phi$ be a polynomially growing outer automorphism of $\mathbf{F}_{r}$. Up to replacement of $\Phi$ with a power of $\Phi$, one of the following assertion holds.

(i) There exist $\varphi \in$ Aut $\left(\mathbf{F}_{r}\right)$ representing $\Phi$ and a non-trivial free decomposition $F_{1} * F_{2}$ of $\mathbf{F}_{r}$ which is invariant under $\varphi$.

(ii) There exist $\varphi \in$ Aut $\left(\mathbf{F}_{r}\right)$ representing $\Phi$, a non-trivial free decomposition $F_{1} *\langle t\rangle$ of $\mathbf{F}_{r}$ and an element $f$ of $F_{1}$ such that $F_{1}$ is invariant under $\varphi$ and $\varphi(t)=t f$.

Theorem 1.6. Let $r \geqslant 1$. Let $\Phi$ be a polynomially growing outer automorphism of $\mathbf{F}_{r}$. For all positive integers $n, \Phi$ induces an outer automorphism of finite order of $\mathbf{B}_{r}(n)$.

Proof. The proof is by induction on the rank $r$ of the free group. The outer automorphism group of $\mathbb{Z}$ is reduced to $\{\mathrm{id},-\mathrm{id}\}$. Hence the theorem holds for rank one. Let $r \geqslant 1$. Assume that the theorem holds for any rank smaller or equal to $r$. Let $\Phi$ be a polynomially growing outer automorphism of $\mathbf{F}_{r+1}$ and $n$ a positive integer. Following Proposition 1.5, we distinguish two cases.

First case. There exist an automorphism $\varphi \in$ Aut $\left(\mathbf{F}_{r+1}\right)$ representing a power of $\Phi$ and a non-trivial free decomposition $F_{1} * F_{2}$ of $\mathbf{F}_{r+1}$ invariant under $\varphi$. We denote by $\varphi_{i}$ the restriction of $\varphi$ to $F_{i}$. By induction, there exists an integer $p_{i}$ such that $\varphi_{i}^{p_{i}}$ induces the identity of $F_{i} / F_{i}^{n}$. It follows that $\varphi^{p_{1} p_{2}}$ is trivial in Aut $\left(\mathbf{B}_{r+1}(n)\right)$. Therefore $\Phi$ induces an outer automorphism of finite order of $\mathbf{B}_{r+1}(n)$.

Second case. There exist an automorphism $\varphi \in$ Aut $\left(\mathbf{F}_{r+1}\right)$ representing a power of $\Phi$, a free decomposition $F_{1} *\langle t\rangle$ of $\mathbf{F}_{r+1}$ and an element $f$ of $F_{1}$ such that $F_{1}$ is invariant under $\varphi$ and $\varphi(t)=t f$. We denote by $\varphi_{1}$ the restriction of $\varphi$ to $F_{1}$. By induction, there exists an integer $p_{1}$ such that $\varphi_{1}^{p_{1}}$ induces the identity of $F_{1} / F_{1}^{n}$. On the other hand, for all integers $q, \varphi^{q}(t)$ equals $t f \varphi_{1}(f) \varphi_{1}^{2}(f) \ldots \varphi_{1}^{q-1}(f)$. It follows that the equality below holds in $\mathbf{B}_{r+1}(n)$ :

$$
\varphi^{n p_{1}}(t)=t\left[f \varphi_{1}(f) \varphi_{1}^{2}(f) \ldots \varphi_{1}^{p_{1}-1}(f)\right]^{n}=t .
$$

Hence $\varphi^{n p_{1}}$ is trivial in Aut $\left(\mathbf{B}_{r+1}(n)\right)$. Therefore $\Phi$ induces an outer automorphism of finite order of $\mathbf{B}_{r+1}(n)$.

1.3. Subgroups of Out $\left(B_{\boldsymbol{r}}(\boldsymbol{n})\right)$. We are now interested in relevant subgroups that can be embedded in Out $\left(\mathbf{B}_{r}(n)\right)$. We start with free subgroups. The following result is due to M. Bestvina, M. Feighn and M. Handel

Theorem 1.7 (see [5], Theorem 5.2). Let $r \geqslant 3$. Let $\varphi_{1}$ and $\varphi_{2}$ two automorphisms of $\mathbf{F}_{r}$. We assume that the outer automorphisms induced by $\varphi_{1}$ and $\varphi_{2}$ are irreducible, do not have common powers and neither have a nontrivial periodic conjugacy class. 
There exists an integer $m$ such that $\varphi_{1}^{m}$ and $\varphi_{2}^{m}$ generate a free group. Moreover, the semi-direct product $\mathbf{F}_{r} \rtimes \mathbf{F}_{2}$ defined by $\varphi_{1}^{m}$ and $\varphi_{2}^{m}$ is hyperbolic.

Theorem 1.8. Let $r \geqslant 3$. There exists an integer $n_{0}$ such that for all odd integers $n$ larger than $n_{0}$, Out $\left(\mathbf{B}_{r}(n)\right)$ contains a subgroup which is isomorphic to $\mathbf{F}_{2}$.

Proof. Theorem 1.7 provides a hyperbolic extension of $\mathbf{F}_{r}$ by $\mathbf{F}_{2}$. In other words, $1 \rightarrow \mathbf{F}_{r} \rightarrow \mathbf{F}_{r} \rtimes \mathbf{F}_{2} \rightarrow \mathbf{F}_{2} \rightarrow 1$ is a short exact sequence such that $\mathbf{F}_{r} \rtimes \mathbf{F}_{2}$ is hyperbolic. The result follows from the main theorem.

We are now looking for free abelian subgroups of Out $\left(\mathbf{B}_{r}(n)\right)$. Let $G_{1}$ and $G_{2}$ be two torsion-free groups. We denote by $G$ the free product $G_{1} * G_{2}$. Since $G_{1}$ and $G_{2}$ are torsion-free, so is $G$ (see [32]). Moreover, for all integers $n, G^{n} \cap G_{1}=G_{1}^{n}$.

Lemma 1.9. Let $n$ be an integer. Let $\varphi$ be an automorphism of $G$ which stabilizes the factor $G_{1}$. We assume that $\varphi$ induces an automorphism of finite order of $G / G^{n}$. Then, the restriction of $\varphi$ to $G_{1}$ induces also an automorphism of finite order of $G_{1} / G_{1}^{n}$.

Proof. Since $\varphi$ induces an automorphism of finite order of $G / G^{n}$, there exists $k \in \mathbb{N}^{*}$ such that for all $g \in G, \varphi^{k}(g) g^{-1} \in G^{n}$. However $G_{1}$ is invariant under $\varphi$. Thus for all $g \in G_{1}, \varphi^{k}(g) g^{-1} \in G^{n} \cap G_{1}=G_{1}^{n}$. It follows that the restriction of $\varphi$ to $G_{1}$ induces an automorphism of finite order of $G_{1} / G_{1}^{n}$.

Theorem 1.10. Let $r \geqslant 2$. There exists an integer $n_{0}$ such that for all odd integers $n$ larger than $n_{0}$, Out $\left(\mathbf{B}_{2 r}(n)\right)$ and Out $\left(\mathbf{B}_{2 r+1}(n)\right)$ contain a subgroup which is isomorphic to $\mathbb{Z}^{r}$.

Proof. We denote by $\varphi$ the automorphism of $\mathbf{F}_{2}$ studied in Proposition 1.1. There exists an integer $n_{0}$ such that for all odd integers $n$ larger than $n_{0}, \varphi$ induces an automorphism of infinite order of $\mathbf{B}_{2}(n)$. Let $n$ be an odd integer larger than $n_{0}$. We consider $\mathbf{F}_{2 r}$ as a free product $F_{1} * \cdots * F_{r}$ of $r$ copies of $\mathbf{F}_{2}$. For all $i \in\{1, \ldots, r\}$, we define an automorphism $\varphi_{i}$ of $\mathbf{F}_{2 r}$ as follows.

(i) The restriction of $\varphi_{i}$ to $F_{i}$ is $\varphi$.

(ii) The restriction of $\varphi_{i}$ to any other factor is the identity.

By construction, the $\varphi_{i}$ 's generate an abelian subgroup of Aut $\left(\mathbf{F}_{2 r}\right)$ and all the more of Out $\left(\mathbf{B}_{2 r}(n)\right)$. We now study the relations between the $\varphi_{i}$ 's in Out $\left(\mathbf{B}_{2 r}(n)\right)$. Consider $r$ integers $k_{1}, \ldots, k_{r}$ such that $\psi=\varphi_{1}^{k_{1}} \ldots \varphi_{r}^{k_{r}}$ induces an inner automorphism of $\mathbf{B}_{2 r}(n)$. By Lemma 1.9, $\varphi^{k_{i}}$, which is the restriction of $\psi$ to $F_{i}$, induces an automorphism of finite order of $F_{i} / F_{i}^{n}=\mathbf{B}_{2}(n)$. This forces $k_{i}$ to equal zero. Hence the $\varphi_{i}$ 's generate a subgroup of Out $\left(\mathbf{B}_{2 r}(n)\right)$ which is isomorphic to $\mathbb{Z}^{r}$. For Out $\left(\mathbf{B}_{2 r+1}(n)\right)$ we apply the same argument with the following free factorization: $\mathbf{F}_{2 r+1}=F_{1} * \cdots * F_{r} * \mathbb{Z}$. 


\section{Small cancellation theory}

In this section we will be concerned with the small cancellation theory. We expose the geometrical point of view developed by T. Delzant and M. Gromov in [15] and used in Section 3 to prove the main theorem.

2.1. Hyperbolic spaces. Let $X$ be a proper and geodesic space. The distance between two points $x$ and $x^{\prime}$ of $X$ is denoted by $\left|x-x^{\prime}\right|_{X}$ (or simply $\left|x-x^{\prime}\right|$ ). Although it may not be unique, we denote by $\left[x, x^{\prime}\right]$ a geodesic joining $x$ and $x^{\prime}$. We assume that $X$ is $\delta$-hyperbolic (in the sense of Gromov) i.e. for all $x, y, z, t \in X$,

$$
|x-y|+|z-t| \leqslant \max \{|x-z|+|y-t|,|x-t|+|y-z|\}+2 \delta .
$$

The boundary at infinity of $X$ will be denoted by $\partial X$ (see Chapter 2 of [11]). A subset $Y$ of $X$ is $\alpha$-quasi-convex if every geodesic of $X$ joining two points of $Y$ lies in the $\alpha$-neighbourhood of $Y$, denoted by $Y^{+\alpha}$.

Lemma 2.1 (see [15], Lemma 2.1.5, or [12], Corollary 1.2.2). Let $x, x^{\prime}, y$ and $y^{\prime}$ be four points of $X$. Let $u$ be a point of $\left[x, x^{\prime}\right]$ such that $|u-x|>|x-y|+8 \delta$ and $\left|u-x^{\prime}\right|>\left|x^{\prime}-y^{\prime}\right|+8 \delta$. Then $u$ belongs to the $8 \delta$-neighbourhood of $\left[y, y^{\prime}\right]$.

Proposition 2.2 (see [15], Lemma 2.2.2, or [12], Proposition 1.2.4). Let $Y$ and $Z$ be two $\alpha$-quasi-convex subsets of $X$. For all $A \geqslant 0$

$$
\operatorname{diam}\left(Y^{+A} \cap Z^{+A}\right) \leqslant \operatorname{diam}\left(Y^{+\alpha+10 \delta} \cap Z^{+\alpha+10 \delta}\right)+2 A+20 \delta .
$$

Let $g$ be an isometry of $X$. In order to measure its action on $X$, we define two translation lengths. By the translation length $[g]_{X}$ (or simply $[g]$ ) we mean

$$
[g]=\inf _{x \in X}|g x-x| .
$$

The asymptotic translation length $[g]_{X}^{\infty}\left(\right.$ or simply $[g]^{\infty}$ ) is

$$
[g]^{\infty}=\lim _{n \rightarrow+\infty} \frac{1}{n}\left|g^{n} x-x\right| .
$$

These two lengths satisfy the following inequality (see [11], Chapter 10, Proposition 6.4):

$$
[g]^{\infty} \leqslant[g] \leqslant[g]^{\infty}+32 \delta \text { for all } g \in G .
$$

The axis $A_{g}$ of $g$, defined as follows, is a $40 \delta$-quasi-convex subset of $X$ (see [15], Proposition 2.3.3):

$$
A_{g}=\{x \in X /|g x-x| \leqslant \max \{[g], 40 \delta\}\} .
$$


The isometry $g$ is hyperbolic if its asymptotic translation length is positive. In this case, $g$ fixes exactly two points of $\partial X$ denoted by $g^{-}$and $g^{+}$. The cylinder of $\left\{g^{-}, g^{+}\right\}$, denoted by $Y_{g}$, is defined to be the set of points of $X$ which are $10 \delta$-close to some geodesic joining $g^{-}$and $g^{+}$. It is a $g$-invariant, $8 \delta$-quasi-convex subset of $X$.

Proposition 2.3. Let $g$ be a hyperbolic isometry of $X$. We denote by $\sigma$ a geodesic joining $g^{-}$and $g^{+}$, the points of $\partial X$ fixed by $g$. Let $Y$ be a $\alpha$-quasi-convex subset of $X$. If $Y$ is $g$-invariant, then $\sigma$ is contained in the $(\alpha+8 \delta)$-neighbourhood of $Y$. In particular, $\sigma$ is contained in the 488 -neighbourhood of $A_{g}$.

Proof. Let $x$ be a point of $\sigma$. We write $d$ for the distance between $x$ and $Y$. Let $y$ be a point of $Y$ such that $|x-y| \leqslant d+\delta$. Since $g$ is hyperbolic, there is an integer $m$ such that $\left|g^{m} x-g^{-m} x\right|>2 d+48 \delta$ (see [11], Chapter 10, Lemme 6.5). We denote by $p_{+}$(respectively $p_{-}$) a projection of $g^{m} x$ (respectively $g^{-m} x$ ) on $\sigma$, i.e. a point of $\sigma$ such that $\left|g^{m} x-p_{+}\right|=d\left(g^{m} x, \sigma\right)$ (respectively $\left.\left|g^{-m} x-p_{-}\right|=d\left(g^{-m} x, \sigma\right)\right)$. The geodesics $\sigma$ and $g^{m} \sigma$ have the same extremities. It follows that they are $8 \delta$-close (see [11], Chapter 2, Proposition 2.2). In particular, $\left|g^{m} x-p_{+}\right| \leqslant 8 \delta$. In the same way, we have $\left|g^{-m} x-p_{-}\right| \leqslant 8 \delta$. Note that $x$ lies on the subgeodesic of $\sigma$ delimited by $p_{-}$and $p_{+}$. Indeed, if it was not the case we should have

$$
\begin{aligned}
\left|g^{-m} x-g^{m} x\right| \leqslant\left|p_{-}-p_{+}\right|+16 \delta & \leqslant \| x-p_{-}|-| x-p_{+}||+16 \delta \\
& \leqslant \| x-g^{-m} x|-| x-g^{m} x||+32 \delta \\
& \leqslant 32 \delta .
\end{aligned}
$$

Contradiction. On the other hand, we have

$$
\left|x-p_{+}\right| \geqslant\left|x-g^{m} x\right|-8 \delta \geqslant \frac{1}{2}\left|g^{-m} x-g^{m} x\right|-8 \delta>d+16 \delta .
$$

Hence

$$
\left|x-p_{+}\right|>\left|g^{m} y-g^{m} x\right|+\left|g^{m} x-p_{+}\right|+8 \delta \geqslant\left|g^{m} y-p_{+}\right|+8 \delta .
$$

In the same way, we have $\left|x-p_{-}\right|>\left|g^{-m} y-p_{-}\right|+8 \delta$. By Lemma 2.1, the point $x$ is $8 \delta$-close to $\left[g^{-m} y, g^{m} y\right]$. However $g^{-m} y$ and $g^{m} y$ belong to $Y$ which is $\alpha$-quasi-convex. Therefore the distance between $x$ and $Y$ is smaller than $\alpha+8 \delta$.

Definition 2.4. Let $P$ be a set of isometries of $X$. The injectivity radius denoted by $r_{\text {inj }}(P, X)$ and the maximal overlap denoted by $\Delta(P, X)$ are the following quantities

$$
\begin{gathered}
r_{\text {inj }}(P, X)=\inf \left\{[g]^{\infty} / g \in P^{*}\right\}, \\
\Delta(P, X)=\sup \left\{\operatorname{diam}\left(Y_{g}^{+20 \delta} \cap Y_{g^{\prime}}^{+20 \delta}\right) / g, g^{\prime} \in P^{*}, g \neq g^{\prime}\right\},
\end{gathered}
$$

where $P^{*}$ denotes the set of hyperbolic elements of $P$. 
Let $G$ be a group acting properly, co-compactly, by isometries on $X$. An element $g$ of $G$ is either elliptic (in particular, it has finite order) or hyperbolic (see [11], Chapter 9). A subgroup of $G$ is called elementary if it is virtually cyclic. Since $G$ is a hyperbolic group, every non-elementary subgroup of $G$ contains a copy of $\mathbf{F}_{2}$, the free group of rank 2 (see [16], Chapter 8, Theorem 37). Given a hyperbolic element $g$ of $G$, the normalizer of $\langle g\rangle$ is elementary (see [11], Chapter 10, Corollary 7.2). The group $G$ satisfies the small centralizers hypothesis if $G$ is non-elementary and every elementary subgroup of $G$ is cyclic. The next lemma will be needed in Section 2.5.

Lemma 2.5. We assume that $G$ satisfies the small centralizers hypothesis. Let $R$ be a subset of $G$ stable by conjugation and inverse, whose elements are hyperbolic and not proper powers. There exists a subset $R_{0}$ of $R$, stable by conjugation satisfying the followings:

(i) for all $h \in R$ one and only one element of $\left\{h, h^{-1}\right\}$ belongs to $R_{0}$,

(ii) any two distinct elements of $R_{0}$ generate a non-elementary subgroup.

Proof. To prove (i) it is sufficient to show that an element $h$ of $R$ cannot be conjugate to its inverse. Suppose, contrary to our claim, that there exist $h \in R$ and $g \in G$ such that $g h g^{-1}=h^{-1}$. Thus, $g$ belongs to the normalizer of $h$, which is elementary (see [11], Chapter 10, Proposition 1). In particular, $g$ and $h$ generate an elementary subgroup of $G$. Since $G$ satisfies the small centralizers hypothesis, $g$ and $h$ commute. It follows that $h=h^{-1}$. Hence $h$ is not hyperbolic, a contradiction.

Let us now prove (ii). Let $h_{1}, h_{2} \in R_{0}$. Assume that they generate an elementary subgroup. $G$ satisfying the small centralizers hypothesis, $h_{1}$ and $h_{2}$ commute. Since $h_{1}$ and $h_{2}$ are not proper powers, they are either equal or inverse. However $R_{0}$ does not contain an element and its inverse. Hence $h_{1}=h_{2}$.

Definition 2.6. Let $G$ be a group acting properly co-compactly by isometries on a proper, geodesic, $\delta$-hyperbolic space $X$. The invariant $A(G, X)$ is the upper bound of $\operatorname{diam}\left(A_{g}^{+50 \delta} \cap A_{g^{\prime}}^{+50 \delta}\right)$, where $g$ and $g^{\prime}$ are two elements of $G$ which generate a non-elementary subgroup and whose translation lengths are smaller than $100 \delta$.

Remark. If there is no such $g$ and $g^{\prime}$ we adopt the convention that $A(G, X)=+\infty$. However in our study, $G$ will be non-elementary and the translation lengths of its generators small in comparison to $\delta$. Therefore $A(G, X)$ will always be finite.

This invariant $A(G, X)$ was used by T. Delzant and M. Gromov to study hyperbolic groups satisfying the small centralizers hypothesis (see §2.4 in [15]).

Remark. The objects defined previously depend implicitly on the hyperbolicity constant $\delta$ (e.g. $Y_{g}, A_{g}$, and $A(G, X)$ ). Although the notation does not make this dependency explicit, we should keep in mind that it plays an important role. For instance, we have the following lemma: 
Lemma 2.7. Let $X$ be proper, geodesic, $\delta$-hyperbolic space and $G$ a group acting properly, co-compactly by isometries on $X$. Let $\lambda$ be a positive number. Then $A(G, \lambda X)=\lambda A(G, X)$, where $\lambda X$ is the $\lambda \delta$-hyperbolic space $X$ endowed with the rescaled metric $\lambda|\cdot|_{X}$.

Proof. Let $g$ be an element of $G$. Its translation lengths satisfy $[g]_{\lambda X}=\lambda[g]_{X}$ and $[g]_{\lambda X}^{\infty}=\lambda[g]_{X}^{\infty}$. Since $\lambda X$ is a $\lambda \delta$-hyperbolic space, the axis of $g$ in $\lambda X$ is exactly the image in $\lambda X$ of the axis $A_{g}$ of $g$ in $X$. We will denote it by $\lambda A_{g}$. Let $g$ and $g^{\prime}$ be two elements of $G$ that do not generate an elementary group and whose asymptotic translation lengths in $\lambda X$ are smaller than $100 \lambda \delta$. In particular, we have $[g]_{X}^{\infty},\left[g^{\prime}\right]_{X}^{\infty} \leqslant 100 \delta$. By definition of $A(G, X)$, we have

$$
\begin{aligned}
\operatorname{diam}\left(\lambda A_{g}^{+50 \lambda \delta} \cap \lambda A_{g^{\prime}}^{+50 \lambda \delta}\right) & =\lambda \operatorname{diam}\left(A_{g}^{+50 \delta} \cap A_{g^{\prime}}^{+50 \delta}\right) \\
& \leqslant \lambda A(G, X) .
\end{aligned}
$$

After taking the upper bound for all $g$ and $g^{\prime}$, we obtain $A(G, \lambda X) \leqslant \lambda A(G, X)$. In the same way, $A(G, \lambda X) \geqslant \lambda A(G, X)$. This establishes the desired equality.

2.2. Small cancellation theorem. In the remainder of Section 2 we require $X$ to be a proper, geodesic, simply-connected, $\delta$-hyperbolic space and $G$ a group acting properly, co-compactly, by isometries on $X$.

Let $P$ be a set of hyperbolic elements of $G$. We assume that $P$ is stable by conjugation and contains only a finite number of conjugacy classes. We denote by $N$ the (normal) subgroup of $G$ generated by $P$. Our goal is to study the quotient $\bar{G}=G / N$. The small cancellation parameters $\Delta(P, X)$ and $r_{\text {inj }}(P, X)$, defined in the previous section, respectively play the role of the length of the largest piece and the length of the smallest relation in the usual small cancellation theory. We are interested in situations where the ratios $\frac{\delta}{r_{\text {inj }}(P, X)}$ and $\frac{\Delta(P, X)}{r_{\text {inj }}(P, X)}$ are very small (see Theorem 2.8 below). To that end, we build a space $\bar{X}$ with an action of $\bar{G}$. We only recall the main steps of this construction. For more details we refer the reader to [12] and [13].

Fix $r_{0}>0$. Its value will be made precise in the small cancellation theorem (see Theorem 2.8). Let $\rho \in P$. We endow $Y_{\rho}$ with the length metric $|.|_{\rho}$ induced by the restriction of $|.|_{X}$ to $Y_{\rho}$. The cone over $Y_{\rho}$ denoted by $C_{\rho}\left(r_{0}\right)$ (or simply $C_{\rho}$ ) is the topological quotient of $Y_{\rho} \times\left[0, r_{0}\right]$ by the equivalence relation which identifies the points $(y, 0)$ for all $y \in Y_{\rho}$. Given two points $x=(y, r)$ and $x^{\prime}=\left(y^{\prime}, r^{\prime}\right)$ of $C_{\rho}$ the following formula defines a distance on $C_{\rho}$ (see [7], Chapter I.5, Proposition 5.9 (1)):

$$
\cosh \left(\left|x, x^{\prime}\right|\right)=\cosh r \cosh r^{\prime}-\sinh r \sinh r^{\prime} \cos \left(\min \left\{\pi, \frac{\left|y-y^{\prime}\right|_{\rho}}{\sinh r_{0}}\right\}\right) \text {. }
$$

The cone-off over $X$ relatively to $P$, denoted by $\dot{X}_{P}\left(r_{0}\right)$ (or simply $\dot{X}$ ), is obtained by attaching for all $\rho \in P$ the cone $C_{\rho}$ to $X$ along $Y_{\rho}$. The distances $|\cdot|_{X}$ and $|\cdot|_{C_{\rho}}$ 
induce a metric on $\dot{X}$ (see [12], Proposition 3.1.7). We extend by homogeneity the action of $G$ on $X$ in an action of $G$ on $\dot{X}$. Let $x=(y, r) \in C_{\rho}$ and $g \in G$. We define $g x$ to be the point of $C_{g \rho g^{-1}}=g C_{\rho}$ given by $g x=(g y, r)$. The group $G$ acts by isometries on $\dot{X}$ (see [12], Lemma 4.3.1). The space $\bar{X}_{P}\left(r_{0}\right)$ (or simply $\bar{X}$ ) is the quotient of $\dot{X}$ by $N$.

Theorem 2.8 (Small cancellation theorem, see [15], Theorem 5.5.2, or [12], Theorem 4.2.2). There exist positive numbers $\delta_{0}, \delta_{1}, \Delta_{0}$ and $r_{0} \geqslant 10^{5} \delta_{1}$ satisfying the following properties.

Let $X$ be a proper, geodesic, simply-connected, $\delta$-hyperbolic space. Let $G$ be a group acting properly, co-compactly, by isometries on $X$. Let $P$ be a set of hyperbolic elements of $G$. We assume that $P$ is stable by conjugation and contains only a finite number of conjugacy classes.

If $\delta \leqslant \delta_{0}, \Delta(P, X) \leqslant \Delta_{0}$ and $r_{\text {inj }}(P, X) \geqslant 3 \pi \sinh r_{0}$, then $\bar{X}_{P}\left(r_{0}\right)$ is proper, geodesic, simply-connected and $\delta_{1}$-hyperbolic. Moreover $\bar{G}$ acts properly, co-compactly, by isometries on it .

Remark. The fact that the constants $r_{0}, \delta_{0}, \delta_{1}$ and $\Delta_{0}$ do not depend on $X, P$ or $G$ is very important in order to iterate the small cancellation construction.

2.3. Estimation of an injectivity radius. From now on we suppose that $X, G$ and $P$ satisfy the assumptions of the small cancellation theorem. In particular, $\delta \leqslant \delta_{0}$, $\Delta(P, X) \leqslant \Delta_{0}$ and $r_{\text {inj }}(P, X) \geqslant 3 \pi \sinh r_{0}$, where $\delta_{0}, \Delta_{0}$ and $r_{0}$ are the constants given by Theorem 2.8. We also assume now that $G$ satisfies the small centralizers hypothesis, i.e. $G$ is non-elementary and all elementary subgroups of $G$ are cyclic (see Section 2.1). We are now interested in the properties of the action of $\bar{G}$ on $\bar{X}$. This will allow us to iterate the small cancellation process. We need in particular an estimation of $A(\bar{G}, \bar{X})$ and some injectivity radius. We already know that $\bar{X}$ is $\delta_{1}$-hyperbolic (see Theorem 2.8).

For all $\rho \in P$, we write $E_{\rho}$ for the subgroup of $G$ which stabilizes $Y_{\rho}$. It is an elementary subgroup of $G$ (see [11], Chapter 10, Proposition 7.1). Let $v$ and $\pi$ be the respective maps $v: \dot{X} \rightarrow \bar{X}$ and $\pi: G \rightarrow \bar{G}$. The space $\bar{X}$ is obtained by attaching cones of large radius to $v(X)$. This provides a kind of Margulis' decomposition. The cones play the role of the thick part: the translation length of a hyperbolic element of $\bar{G}$ in a cone is very large. The following lemma illustrates this fact.

Lemma 2.9 (see [15], Lemme 5.9.3). Let $\bar{g}$ be an element of $\bar{G}$ such that $[\bar{g}] \leqslant 200 \delta_{1}$. Assume that $\bar{g}$ does not belong to $\bar{E}_{\rho}=\pi\left(E_{\rho}\right)$ for all $\rho \in P$. Then $A_{\bar{g}}$ is contained in $v(X)^{+100 \delta_{1}}$ and $A_{\bar{g}} \cap v(X)$ is non-empty.

To study $v(X)^{+100 \delta_{1}}$, which is an analogue of the thin part of the Margulis decomposition, we use the fact that the map $v(X) \rightarrow \bar{X}$ is a local quasi-isometry: 
Lemma 2.10 (see [12], Proposition 3.1.8). Let $x$ and $x^{\prime}$ be two points of $X$. First $\left|x-x^{\prime}\right|_{\dot{X}} \leqslant\left|x-x^{\prime}\right|_{X}$. Moreover, if $\left|x-x^{\prime}\right|_{\dot{X}} \leqslant \frac{r_{0}}{2}$ then $\left|x-x^{\prime}\right|_{X} \leqslant \frac{2 \pi \sinh r_{0}}{r_{0}}\left|x-x^{\prime}\right|_{\dot{X}}$.

Using this point of view, T. Delzant and M. Gromov proved the following result.

Proposition 2.11 (see [15], Lemme. 5.10.1). Let $\bar{C}$ be a $50 \delta_{1}$-quasi-convex subset of $v(X)^{+100 \delta_{1}}$. There exists a subset $C$ of $\dot{X}$ such that

(i) the map $v: \dot{X} \rightarrow \bar{X}$ induces an isometry from $C$ onto $\bar{C}$,

(ii) the projection $\pi: G \rightarrow \bar{G}$ induces an isomorphism between $\operatorname{Stab}(C)$ and $\operatorname{Stab}(\bar{C})$ which are respectively the stabilizers of $C$ and $\bar{C}$.

Proposition 2.12. Let $H$ be a normal subgroup of $G$ containing $P$ and $\bar{H}$ its image by the projection $\pi: G \rightarrow \bar{G}$. Then $r_{\mathrm{inj}}(\bar{H}, \bar{X}) \geqslant \min \left\{\kappa l, \delta_{1}\right\}$, where $\kappa$ stands for $\frac{3 r_{0}}{40 \pi \sinh r_{0}}$ and $l$ is the smallest asymptotic translation length of a hyperbolic element of $H$ that does not belong to any $E_{\rho}$.

Remark. This lemma is a refinement of Lemma 5.11.1 proved by T. Delzant and M. Gromov in [15]. They gave indeed a lower bound for $r_{\text {inj }}(\bar{G}, \bar{X})$. Four our purpose, we need a more accurate result. We provide here an estimation of the injectivity radius of a normal subgroup of $\bar{G}$.

Proof. Since $H$ is a normal subgroup containing the relations $P, \pi^{-1}(\bar{H})=H$. Suppose the assertion of the lemma is false. There exists a hyperbolic element $\bar{h}$ of $\bar{H}$ such that $[\bar{h}]^{\infty}<\min \left\{\kappa l, \delta_{1}\right\}$. Fix an integer $m$ such that $7 \delta_{1} \leqslant m \min \left\{\kappa l, \delta_{1}\right\} \leqslant$ $8 \delta_{1}$. By [11], Chapter 10, Proposition 6.4,

$$
\left[\bar{h}^{m}\right] \leqslant m[\bar{h}]^{\infty}+32 \delta_{1}<m \min \left\{\kappa l, \delta_{1}\right\}+32 \delta_{1} \leqslant 40 \delta_{1} .
$$

Since $\left[h^{m}\right] \leqslant 200 \delta_{1}$, the axis $A_{\bar{h}^{m}}$ of $\bar{h}^{m}$ in $\bar{X}$, is a $40 \delta_{1}$-quasi-convex (see [15], Proposition 2.3.3), which is contained in the $100 \delta_{1}$-neighbourhood of $v(X)$ (see Lemma 2.9). By Proposition 2.11, there exists a subset $C$ of $X$ such that

(i) the map $v: \dot{X} \rightarrow \bar{X}$ induces an isometry from $C$ onto $A_{\bar{h}^{m}}$,

(ii) the map $\pi: G \rightarrow \bar{G}$ induces an isomorphism from $\operatorname{Stab}(C)$ onto $\operatorname{Stab}\left(A_{\bar{h}^{m}}\right)$.

However $\bar{h}$ belongs to $\operatorname{Stab}\left(A_{\bar{h}^{m}}\right)$. We denote by $h$ its preimage in $\operatorname{Stab}(C)$. Since $\bar{h}$ is hyperbolic, $h$ is necessarily a hyperbolic element of $H$ that does not belong to $E_{\rho}$ for all $\rho \in P$. By assumption, $[h]_{X}^{\infty} \geqslant l$.

On the other hand by Lemma 2.9, $A_{\bar{h}^{m}} \cap v(X) \neq \emptyset$. Fix $\bar{x} \in A_{\bar{h}^{m}} \cap v(X)$ and denote by $x$ its preimage in $C \cap X$. As the map $v: C \rightarrow A_{\bar{h}^{m}}$ is an equivariant isometry we have $\left|h^{m} x-x\right|_{\dot{X}}=\left|\bar{h}^{m} \bar{x}-\bar{x}\right|_{\bar{X}}$. Recall that $\bar{x}$ belongs to $A_{\bar{h}^{m}}$, the axis of $\bar{h}^{m}$. It follows that

$$
\left|h^{m} x-x\right|_{\dot{X}}=\left|\bar{h}^{m} \bar{x}-\bar{x}\right|_{\bar{X}} \leqslant \max \left\{\left[\bar{h}^{m}\right], 40 \delta_{1}\right\} \leqslant 40 \delta_{1} .
$$


By Lemma 2.10, $\left|h^{m} x-x\right|_{X}$ is smaller than $\frac{2 \pi \sinh r_{0}}{r_{0}}\left|h^{m} x-x\right|_{\dot{X}}$. Consequently,

$$
m l \leqslant m[h]_{X}^{\infty} \leqslant\left[h^{m}\right]_{X} \leqslant\left|h^{m} x-x\right|_{X} \leqslant \frac{80 \pi \delta_{1} \sinh r_{0}}{r_{0}}=\frac{6 \delta_{1}}{\kappa} .
$$

In particular, $7 \delta_{1} \leqslant m \min \left\{\kappa l, \delta_{1}\right\} \leqslant m \kappa l \leqslant 6 \delta_{1}$, a contradiction.

2.4. Other properties of $\bar{G}$ and $\bar{X}$. In this section, we review some results obtained by T. Delzant and M. Gromov in [15].

Proposition 2.13 (see [15], Lemme 5.9.5). The constant $A(\bar{G}, \bar{X})$ satisfies the following inequality.

$$
A(\bar{G}, \bar{X}) \leqslant A(G, X)+1000 \delta_{1} e^{350 \delta_{1}} .
$$

Proposition 2.14 (see [15], Lemme 5.10.2 and Lemme 5.10.3). Assume that every element of $P$ is an odd power of an element of $G$ which is not a proper power. Then $\bar{G}$ satisfies the following properties.

(i) Every elementary subgroup of $\bar{G}$ is cyclic.

(ii) Let $\bar{F}$ be a finite subgroup of $\bar{G}$. Either $\bar{F}$ is the image of a finite subgroup of $G$, or there exists $\rho \in P$ such that $\bar{F}$ is a subgroup of $\bar{E}_{\rho}=\pi\left(E_{\rho}\right)$.

Proposition 2.15 (see [15], Theorem 5.7.1). The Euler characteristic of $\bar{G}$ satisfies $\chi(\bar{G}, \mathbb{Q})=\chi(G, \mathbb{Q})+|P / G|$, where $|P / G|$ denotes the number of conjugacy classes of $P$.

2.5. An induction lemma. One should think of the next lemma as a step of the iterative procedure involved in the proof of the main theorem. We recall that the invariant $A(G, X)$ stands for the maximal overlap between the axes of two small hyperbolic elements of $G$ (see Definition 2.6). The injectivity radius $r_{\text {inj }}(H, X)$ denotes the smallest asymptotic translation length of a hyperbolic element of $H$ (see Definition 2.4).

Lemma 2.16 (Induction lemma). There exist positive numbers $\delta_{1}, \Delta_{1}, l_{1}, l_{2}, l_{3}$ and an integer $n_{0}$ satisfying the following properties. Let $n$ be an odd integer larger than $n_{0}$. Let $X$ be a proper, geodesic, simply-connected, $\delta_{1}$-hyperbolic space. Let $G$ be a group acting properly, co-compactly, by isometries on $X$ and $H$ a normal subgroup of $G$. Let $R$ be the set of hyperbolic elements of $H$, which are not proper powers in $G$ and whose asymptotic translation lengths are smaller than $l_{1}$. Let $N$ be the normal subgroup of $G$ generated by $\left\{h^{n} / h \in R\right\}, \bar{G}$ the quotient $G / N$ and $\bar{H}$ the image of $H$ by the canonical map $\pi: G \rightarrow \bar{G}$. We assume that

(i) G satisfies the small centralizers hypothesis; moreover the order of every finite subgroup of $G$ divides $n$, 
(ii) $A(G, X) \leqslant \Delta_{1}$ and $r_{\mathrm{inj}}(H, X) \geqslant \frac{l_{2}}{\sqrt{n}}$,

(iii) $\chi(G, \mathbb{Q})+\frac{1}{2}|R / G|>0$, where $\chi(G, \mathbb{Q})$ is the Euler characteristic of $G$ and $|R / G|$ the number of conjugacy classes in $R$.

Then, there exists a proper, geodesic, simply-connected, $\delta_{1}$-hyperbolic space $\bar{X}$ on which $\bar{G}$ acts properly, co-compactly, by isometries. Moreover, $\bar{G}, \bar{H}$ and $\bar{X}$ satisfy the points (i) and (ii), $\chi(\bar{G}, \mathbb{Q})>0$ and

$$
[\pi(g)]_{\bar{X}}^{\infty} \pi(g) \leqslant \frac{l_{3}}{\sqrt{n}}[g]_{X}^{\infty} \text { for all } g \in G .
$$

Remark. If $G, H, X$ and $n$ satisfy the hypothesis of the previous lemma-including hypotheses (i), (ii) and (iii) - we will say that $(G, H, X)$ satisfies the induction assumptions for exponent $n$. The induction lemma says in particular that if $(G, H, X)$ satisfies the induction assumptions for exponent $n$, so does $(\bar{G}, \bar{H}, \bar{X})$.

The remainder of this section will be devoted to the proof of the induction lemma.

Proof. The positive numbers $r_{0}, \delta_{0}, \delta_{1}$, and $\Delta_{0}$ are given by the small cancellation theorem (see Theorem 2.8). The constant $\kappa=\frac{3 r_{0}}{40 \pi \sinh r_{0}}$ is the one that appears in Proposition 2.12. We define a rescaling parameter $L_{n}=\sqrt{\frac{n \kappa \delta_{1}}{\pi \sinh r_{0}}}$. The sequence $\left(L_{n}\right)$ is increasing and tends to infinity. Up to chose $n_{0}$ large enough, we may assume that for all $n \geqslant n_{0}$

$$
\begin{aligned}
\frac{\delta_{1}}{L_{n}} & \leqslant \delta_{0}, \\
\frac{2000 \delta_{1} e^{350 \delta_{1}}+176 \delta_{1}}{L_{n}} & \leqslant \min \left\{\Delta_{0}, 1000 \delta_{1} e^{350 \delta_{1}}\right\}, \\
\frac{3 \kappa \delta_{1}}{L_{n}} & \leqslant \delta_{1} .
\end{aligned}
$$

Note that $n_{0}$ only depends on $\delta_{0}, \delta_{1}, \Delta_{0}$ and $r_{0}$. We now define the following constants:

$$
\Delta_{1}=2000 \delta_{1} e^{350 \delta_{1}}, \quad l_{1}=3 \delta_{1}, \quad l_{2}=3 \sqrt{\kappa \delta_{1} \pi \sinh r_{0}} \quad \text { and } \quad l_{3}=\sqrt{\frac{\pi \sinh r_{0}}{\kappa \delta_{1}}} .
$$

Let $n$ be an odd integer larger than $n_{0}$. We assume that $(G, H, X)$ satisfies the induction assumptions for exponent $n$. In particular, $R$ is the set of hyperbolic elements of $H$, which are not a proper powers in $G$ and whose asymptotic translation lengths are smaller than $l_{1}$. For the remainder of the proof of Lemma 2.16 we consider the action of $G$ on the rescaled space $\frac{1}{L_{n}} X$. By (1), this space is $\delta$-hyperbolic with 
$\delta=\frac{\delta_{1}}{L_{n}} \leqslant \delta_{0}$. As explained in the remark on page 798, we should keep in mind that the hyperbolicity constant we are now always working with is $\delta$.

By Lemma 2.5, there exists a subset $R_{0}$ of $R$, stable by conjugation satisfying the followings:

(i) for all $h \in R$ one and only one element of $\left\{h, h^{-1}\right\}$ belongs to $R_{0}$,

(ii) any two distinct elements of $R_{0}$ generate a non-elementary subgroup.

Let us denote by $P$ the set of relations $P=\left\{h^{n}, h \in R_{0}\right\}$.

Lemma 2.17. The set $P$ satisfies the following assumptions:

$$
\Delta\left(P, \frac{1}{L_{n}} X\right) \leqslant \Delta_{0} \text { and } r_{\text {inj }}\left(P, \frac{1}{L_{n}} X\right) \geqslant 3 \pi \sinh r_{0} .
$$

Proof. Let $h_{1}$ and $h_{2}$ be two elements of $R_{0}$ such that $h_{1}^{n} \neq h_{2}^{n}$. By Proposition 2.3, $Y_{h_{j}^{n}}$ is contained in $A_{h_{j}}^{+58 \delta}$. Thus Proposition 2.2 gives

$$
\begin{aligned}
\operatorname{diam}\left(Y_{h_{1}^{n}}^{+20 \delta} \cap Y_{h_{2}^{n}}^{+20 \delta}\right) & \leqslant \operatorname{diam}\left(A_{h_{1}}^{+78 \delta} \cap A_{h_{2}}^{+78 \delta}\right) \\
& \leqslant \operatorname{diam}\left(A_{h_{1}}^{+50 \delta} \cap A_{h_{2}}^{+50 \delta}\right)+176 \delta
\end{aligned}
$$

If $h_{1}$ and $h_{2}$ generated an elementary subgroup, by Lemma 2.5 we should have $h_{1}^{n}=h_{2}^{n}$. Hence $h_{1}$ and $h_{2}$ generate a non-elementary subgroup. On the other hand, $\left[h_{1}\right]^{\infty}$ and $\left[h_{2}\right]^{\infty}$ are smaller than $\frac{l_{1}}{L_{n}}=\frac{3 \delta_{1}}{L_{n}}=3 \delta$. By definition of $A(G, X)$,

$$
\begin{aligned}
\operatorname{diam}\left(Y_{h_{1}^{n}}^{+20 \delta} \cap Y_{h_{2}^{n}}^{+20 \delta}\right) & \leqslant A\left(G, \frac{1}{L_{n}} X\right)+176 \delta \\
& \leqslant \frac{1}{L_{n}} A(G, X)+176 \delta \\
& \leqslant \frac{\Delta_{1}+176 \delta_{1}}{L_{n}}=\frac{2000 \delta_{1} e^{350 \delta_{1}}+176 \delta_{1}}{L_{n}} .
\end{aligned}
$$

By (2), $\Delta\left(P, \frac{1}{L_{n}} X\right)$ is smaller than $\Delta_{0}$.

The injectivity radius of $H$ on $\frac{1}{L_{n}} X$ is larger than

$$
\frac{1}{L_{n}} r_{\text {inj }}(H, X) \geqslant \frac{1}{L_{n}} \frac{l_{2}}{\sqrt{n}}=\sqrt{\frac{\pi \sinh r_{0}}{n \kappa \delta_{1}}} \frac{3 \sqrt{\kappa \delta_{1} \pi \sinh r_{0}}}{\sqrt{n}}=\frac{3 \pi \sinh r_{0}}{n}
$$

In particular, $\left[h^{n}\right]^{\infty} \geqslant 3 \pi \sinh r_{0}$ for all $h \in R_{0}$. Therefore, $r_{\text {inj }}\left(P, \frac{1}{L_{n}} X\right) \geqslant$ $3 \pi \sinh r_{0}$. 
On account of the previous lemma, we can now apply the small cancellation theorem (Theorem 2.8) to the rescaled space $\frac{1}{L_{n}} X, G$ and $P$. We obtain a proper, geodesic, simply-connected, $\delta_{1}$-hyperbolic space $\bar{X}$ on which $\bar{G}=G / \ll P \gg$ acts properly, co-compactly, by isometries.

Lemma 2.18. Every elementary subgroup of $\bar{G}$ is cyclic, either infinite or finite with order dividing $n$.

Proof. All elements of $P$ are odd powers of elements of $G$ which are not proper powers. By Proposition 2.14, all elementary subgroups of $\bar{G}$ are cyclic. Assume now that $\bar{F}$ is a finite subgroup of $\bar{G}$. According to the same proposition, we distinguish two cases.

(i) $\bar{F}$ is the image of a finite subgroup of $G$. However, the order of every finite subgroup of $G$ divides $n$. Thus the order of $\bar{F}$ divides $n$.

(ii) There exists $h \in R_{0}$ such that $\bar{F}$ is a subgroup of $\bar{E}_{h^{n}}=\pi\left(E_{h^{n}}\right)=\langle\pi(h)\rangle$, whose order divides $n$.

Lemma 2.19. The constant $A(\bar{G}, \bar{X})$ is bounded above by $\Delta_{1}$. The injectivity radius $r_{\text {inj }}(\bar{H}, \bar{X})$ is bounded below by $\frac{l_{2}}{\sqrt{n}}$.

Proof. By Proposition 2.13, $A(\bar{G}, \bar{X}) \leqslant A\left(G, \frac{1}{L_{n}} X\right)+1000 \delta_{1} e^{350 \delta_{1}}$. However, using inequality (2), we obtain

$$
A\left(G, \frac{1}{L_{n}} X\right) \leqslant \frac{1}{L_{n}} A(G, X) \leqslant \frac{\Delta_{1}}{L_{n}}=\frac{2000 \delta_{1} e^{350 \delta_{1}}}{L_{n}} \leqslant 1000 \delta_{1} e^{350 \delta_{1}} .
$$

Hence $A(\bar{G}, \bar{X}) \leqslant 2000 \delta_{1} e^{350 \delta_{1}}=\Delta_{1}$.

Let $g$ be a hyperbolic element of $H$, which does not belong to $E_{h^{n}}=\langle h\rangle$ for all $h \in R_{0}$. Its asymptotic translation length in $\frac{1}{L_{n}} X$ is larger than $\frac{l_{1}}{L_{n}}=\frac{3 \delta_{1}}{L_{n}}$. By Proposition 2.12 and inequality (3),

$$
r_{\text {inj }}(\bar{H}, \bar{X}) \geqslant \min \left\{\frac{3 \kappa \delta_{1}}{L_{n}}, \delta_{1}\right\}=\frac{3 \kappa \delta_{1}}{L_{n}}=\frac{3 \sqrt{\kappa \delta_{1} \pi \sinh r_{0}}}{\sqrt{n}}=\frac{l_{2}}{\sqrt{n}} .
$$

Lemma 2.20. The Euler characteristic of $\bar{G}$ satisfies

$$
\chi(\bar{G}, \mathbb{Q})=\chi(G, \mathbb{Q})+\frac{1}{2}|R / G|>0
$$

where $|R / G|$ is the number of conjugacy classes of $R$. In particular, $\bar{G}$ is non-elementary. 
Proof. Consider now $h_{1}, h_{2} \in R_{0}$ and $u \in G$ such that $h_{1}^{n}=u h_{2}^{n} u^{-1}$. Since $R_{0}$ is stable by conjugation, $h_{1}$ and $u h_{2} u^{-1}$ are two elements of $R_{0}$ which generate an elementary subgroup. By Lemma 2.5, $h_{1}=u h_{2} u^{-1}$. In other words, there are as many conjugacy classes in $P$ as in $R_{0}$. By construction, there are twice as many conjugacy classes in $R$ as in $R_{0}$. Thus $|P / G|=\left|R_{0} / G\right|=\frac{1}{2}|R / G|$. The result follows from Proposition 2.15 .

Lemma 2.21. For all $g \in G$, we have $\left.[\pi(g)]_{\bar{X}}\right]^{\infty} \leqslant \frac{l_{3}}{\sqrt{n}}[g]_{X}^{\infty}$.

Proof. By Lemma 2.10, the map $\frac{1}{L_{n}} X \rightarrow \bar{X}$ contracts the distances. Thus for all $g \in G$,

$$
[\pi(g)]_{\bar{X}}^{\infty} \leqslant \frac{1}{L_{n}}[g]_{X}^{\infty}=\sqrt{\frac{\pi \sinh r_{0}}{n \kappa \delta_{1}}}[g]_{X}^{\infty}=\frac{l_{3}}{\sqrt{n}}[g]_{X}^{\infty} .
$$

The previous lemma completes the proof of the induction lemma.

\section{Proof of the main theorem}

Recall the statement of the main theorem.

Main theorem. Let $1 \rightarrow H \rightarrow G \rightarrow F \rightarrow 1$ be a short exact sequence of groups. Assume that $H$ is non-trivial, finitely generated, $G$ is hyperbolic, non-elementary, torsion-free and $F$ is torsion-free. There exists an integer $n_{0}$ such that for all odd integers $n$ larger than $n_{0}$, the canonical map $F \rightarrow$ Out $(H)$ induces an injective homomorphism $F \hookrightarrow$ Out $\left(H / H^{n}\right)$.

Proof. The constants $\delta_{1}, \Delta_{1}, l_{1}, l_{2}, l_{3}$ and $n_{0}$ are given by the induction lemma (see Lemma 2.16). Up to increase $n_{0}$, we may also assume that $\frac{l_{3}}{\sqrt{n_{0}}}<1$. Let $1 \rightarrow H \rightarrow G \rightarrow F \rightarrow 1$ be a short exact sequence of groups, which satisfies the hypotheses of the theorem. The strategy is to build by induction a family of short exact sequences $1 \rightarrow H_{k} \rightarrow G_{k} \rightarrow F \rightarrow 1$ with an action of $G_{k}$ on a hyperbolic space $X_{k}$, such that the direct limit $\underset{\lim }{\longrightarrow} H_{k}$ is the Burnside group $H / H^{n}$.

Initialization. Put $H_{0}=H$ and $G_{0}=G$. Let $X_{0}$ be a proper, geodesic, simplyconnected, hyperbolic space on which $G$ acts properly, co-compactly, by isometries. Take for instance the Rips polyhedron of $G$ (see Chapter 5 in [11]). We can assume, by rescaling $X_{0}$ if necessary, that

- $X_{0}$ is $\delta_{1}$-hyperbolic,

- $A\left(G_{0}, X_{0}\right) \leqslant \Delta_{1}$ (see Lemma 2.7), 
- $\left\{h \in H /[h]^{\infty} \leqslant l_{1}, h\right.$ is not a proper power in $\left.G\right\}$ contains a number of conjugacy classes in $G$ bounded below by $-2 \chi(G, \mathbb{Q})$.

The group $G$ is hyperbolic and torsion free. Since $H$ is a non-trivial subgroup of $G$, it contains a hyperbolic element. In particular, the injectivity radius of $H$ is positive (see [14]). Thus, up to increase one more time $n_{0}$, we may assume that $r_{\text {inj }}\left(H_{0}, X_{0}\right) \geqslant \frac{l_{2}}{\sqrt{n_{0}}}$. Hence $\left(G_{0}, H_{0}, X_{0}\right)$ satisfies the induction assumptions for exponent $n_{0}$.

Let $n$ be an odd integer larger than $n_{0} . \quad\left(G_{0}, H_{0}, X_{0}\right)$ satisfies a fortiori the induction assumptions for exponent $n$.

Induction. Let $\left(G_{k}, H_{k}, X_{k}\right)$ satisfying the induction assumptions for exponent $n$. We denote by $R_{k}$ the set of hyperbolic elements of $H_{k}$ which are not proper powers in $G_{k}$ and whose asymptotic translation lengths are smaller than $l_{1}$. Let $N_{k}$ be the normal subgroup of $G_{k}$ generated by $\left\{h^{n} / h \in R_{k}\right\}, G_{k+1}$ the quotient $G_{k} / N_{k}$ and $H_{k+1}$ the image of $H_{k}$ by the canonical map $\pi_{k}: G_{k} \rightarrow G_{k+1}$. By the induction lemma, there exists a metric space $X_{k+1}$ such that $\left(G_{k+1}, H_{k+1}, X_{k+1}\right)$ satisfies the induction assumptions for the exponent $n$. In this way, we obtain two sequences of groups $\left(H_{k}\right)$ and $\left(G_{k}\right)$ whose properties we want to study now.

\section{Properties of $H_{k}$ and $G_{k}$}

Lemma 3.1. For every integer $k$, there exists a map $G_{k} \rightarrow F$ such that the following diagram is commutative. Moreover its rows are short exact sequences.

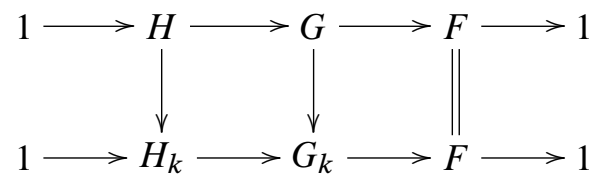

Proof. Following the construction of $H_{k}$ and $G_{k}$, the proof is by induction on $k$. The result is obvious for $k=0$. Assume the lemma holds for $k$. The group $N_{k}$ being generated by elements of $H_{k}, N_{k} \subset H_{k}$. Consequently, the short exact sequence $1 \rightarrow H_{k} \rightarrow G_{k} \rightarrow F \rightarrow 1$ induces maps such that the following diagram commutes and its rows are short exact sequences.

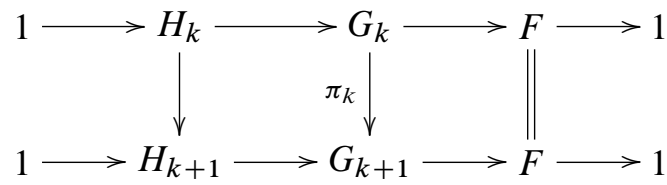

Thus the lemma holds for $k+1$. 
Our next goal is to compare the groups $H / H^{n}$ and $\lim H_{k}$. To shorten notations, we let $h$ stand for an element of $H$ as well as its images in $H_{k}, \underset{\longrightarrow}{\lim } H_{k}$ or $H / H^{n}$.

Lemma 3.2. The kernel of the canonical map $H \rightarrow \underset{\lim }{\longrightarrow} H_{k}$ is exactly $H^{n}$, the subgroup of $H$ generated by all $n$-th powers.

Proof. Let $h$ be an element of $H \backslash\{1\}$. By the induction lemma,

$$
[h]_{X_{k}}^{\infty} \leqslant\left(\frac{l_{3}}{\sqrt{n}}\right)^{k}[h]_{X_{0}}^{\infty} \leqslant\left(\frac{l_{3}}{\sqrt{n_{0}}}\right)^{k}[h]_{X_{0}}^{\infty}
$$

for all integers $k$. However, we chose $n_{0}$ in such a way that $\frac{l_{3}}{\sqrt{n_{0}}}<1$. It follows that there is an integer $k$ such that $[h]_{X_{k}}^{\infty}<\frac{l_{2}}{\sqrt{n}}$. By construction, the injectivity radius of $H_{k}$ on $X_{k}$ is larger than $\frac{l_{2}}{\sqrt{n}}$ (point (ii) of Lemma 2.16). Therefore $h$ is an elliptic isometry. In particular, $h$ has finite order dividing $n$ in $H_{k}$ (point (i) of Lemma 2.16). Hence $H^{n}$ is contained in the kernel of $H \rightarrow \underset{\lim }{\longrightarrow} H_{k}$.

On the other hand, at each step of the construction, the kernel of the map $H_{k} \rightarrow$ $H_{k+1}$ is generated by $n$-th powers of elements of $H_{k}$. It follows that the kernel of the morphism $H \rightarrow \underset{\lim }{\longrightarrow} H_{k}$ is contained in $H^{n}$.

Lemma 3.3. The groups $H / H^{n}$ and $\underline{\lim } H_{k}$ are isomorphic.

Proof. This lemma follows from the previous one and from the fact that the map $H \rightarrow \underset{\lim }{\longrightarrow} H_{k}$ is onto.

Lemma 3.4. Let $f$ be a non-trivial element of $F$. Let $g$ be a preimage of $f$ by the map $G \rightarrow F$. The conjugation by $g$ defines an automorphism of $H$ which induces $a$ non-trivial outer automorphism of $\mathrm{H} / \mathrm{H}^{n}$.

Proof. Let $S$ be a finite generating set of $H$. We denote by $\varphi$ the automorphism of $H$ defined by $\varphi(h)=g h g^{-1}$ for all $h \in H$. Assume, contrary to our claim, that $\varphi$ induces an inner automorphism of $H / H^{n}$. There exists $l \in H$ such that for all $h \in H$ $\varphi(h)$ and $l h l^{-1}$ have the same image in $H / H^{n}$. According to Lemma 3.3, H/H $H^{n}$ and $\lim H_{k}$ are isomorphic. Since $S$ is finite, there is an integer $k$ such that for all $s \in S$ $\overrightarrow{\varphi(s)}$ equals $l s l^{-1}$ in $H_{k}$. However $S$ is a generating set of $H$. Thus $\varphi(h)=g h g^{-1}$ is equal to $l h l^{-1}$ in $H_{k}$ for all $h \in H$. Lemma 3.1 yields the following commutative diagram.

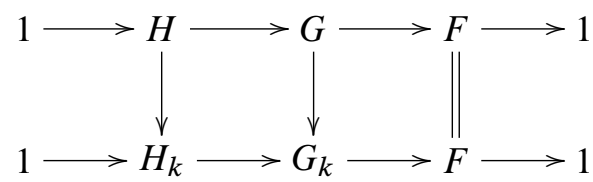


Hence, the image of $l^{-1} g$ in $G_{k}$ commutes with every element of $H_{k}$. By construction, the injectivity radius of $H_{k}$ on $X_{k}$ is positive. Therefore, it contains a hyperbolic element $h$. Thus $h$ and $l^{-1} g$ generate an abelian subgroup of $G_{k}$, which has to be cyclic. There exists $(p, q) \in \mathbb{Z}^{*} \times \mathbb{Z}$ such that $\left(l^{-1} g\right)^{p}=h^{q}$ in $G_{k}$. Using the commutative diagram, we push this identity in $F$ and obtain $f^{p}=1$. Since $F$ is torsion-free, $f$ is trivial. This contradicts our assumption.

End of the proof of the main theorem. The map $F \rightarrow$ Out $(H)$ can be constructed as follows. Let $f$ be an element of $F$ and $g$ a preimage of $f$ by $G \rightarrow F$. The image of $f$ by the map $F \rightarrow$ Out $(H)$ is exactly the outer automorphism of $H$ induced by the conjugation by $g$ in $G$. The previous lemma is hence an equivalent formulation of the following fact: the map $F \rightarrow$ Out $(H)$ induces an injective homomorphism $F \hookrightarrow$ Out $\left(H / H^{n}\right)$. This remark completes the proof of the main theorem.

\section{References}

[1] S. I. Adian, The Burnside problem and identities in groups. Translated from the Russian by John Lennox and James Wiegold, Ergeb. Math. Grenzgeb. 95, Springer-Verlag, Berlin 1979. Zbl 0417.20001 MR 0537580

[2] S. I. Adian and I. G. Lysenok, The method of classification of periodic words and the Burnside problem. In Proceedings of the International Conference on Algebra, Part 1 (Novosibirsk, 1989), Contemp. Math. 131, Amer. Math. Soc., Providence, RI, 1992, 1328. Zbl 0796.20030 MR 1175759

[3] M. Bestvina and M. Feighn, A combination theorem for negatively curved groups. $J$. Differential Geom. 35 (1992), no. 1, 85-101. Zbl 0724.57029 MR 1152226

[4] M. Bestvina and M. Feighn, Addendum and correction to: "A combination theorem for negatively curved groups”. J. Differential Geom. 43 (1996), no. 4, 783-788. Zbl 0862.57027 MR 1412684

[5] M. Bestvina, M. Feighn, and M. Handel, Laminations, trees, and irreducible automorphisms of free groups. Geom. Funct. Anal. 7 (1997), no. 2, 215-244. Zbl 0884.57002 MR 1445386

[6] M. Bestvina, M. Feighn, and M. Handel, Erratum to: "Laminations, trees, and irreducible automorphisms of free groups". Geom. Funct. Anal. 7 (1997), no. 6, 1143. MR 1487756

[7] M. R. Bridson and A. Haefliger, Metric spaces of non-positive curvature. Grundlehren Math. Wiss. 319, Springer-Verlag, Berlin 1999. Zbl 0988.53001 MR 1744486

[8] P. Brinkmann, Hyperbolic automorphisms of free groups. Geom. Funct. Anal. 10 (2000), no. 5, 1071-1089. Zbl 0970.20018 MR 1800064

[9] W. Burnside, On an unsettled question in the theory of discontinuous groups. Quart. $J$. Math. 33 (1902), 230-238. JFM 33.0149.01

[10] E. A. Cherepanov, Free semigroup in the group of automorphisms of the free Burnside group. Comm. Algebra 33 (2005), no. 2, 539-547. Zbl 1121.20028 MR 2124343 
[11] M. Coornaert, T. Delzant, and A. Papadopoulos, Géométrie et théorie des groupes. Les groupes hyperboliques de Gromov, Lecture Notes in Math. 1441, Springer-Verlag, Berlin 1990. Zbl 0727.20018 MR 1075994

[12] R. Coulon, Asphericity and small cancellation theory for rotation family of groups. Groups Geom. Dyn. 5 (2011), no. 4, 729-765. Zbl 06017877 MR 2836458

[13] R. Coulon, Automorphismes extérieurs du groupe de Burnside libre. $\mathrm{PhD}$ thesis, Université de Strasbourg, June 2010.

[14] T. Delzant, Sous-groupes distingués et quotients des groupes hyperboliques. Duke Math. J. 83 (1996), no. 3, 661-682. Zbl 0852.20032 MR 1390660

[15] T. Delzant and M. Gromov, Courbure mésoscopique et théorie de la toute petite simplification. J. Topol. 1 (2008), no. 4, 804-836. Zbl 1197.20035 MR 2461856

[16] É. Ghys and P. de la Harpe, La propriété de Markov pour les groupes hyperboliques. In Sur les groupes hyperboliques d'après Mikhael Gromov (Bern, 1988), Progr. Math. 83, Birkhäuser, Boston, Mass., 1990, 165-187. Zbl 0731.20025 MR 1086657

[17] M. Greendlinger, Dehn's algorithm for the word problem. Comm. Pure Appl. Math. 13 (1960), 67-83. Zbl 0104.01903 MR 0124381

[18] M. Greendlinger, On Dehn's algorithms for the conjugacy and word problems, with applications. Comm. Pure Appl. Math. 13 (1960), 641-677. Zbl 0156.01303 MR 0125020

[19] M. Greendlinger, An analogue of a theorem of Magnus. Arch. Math. 12 (1961), 94-96. Zbl 0103.25603 MR 0132099

[20] R. I. Grigorčuk and I. G. Lysenok, Burnside Problem. In The concise handbook of algebra, A. V. Mikhalev and G. F. Pilz (eds.), Kluwer Academic Publishers, Dordrecht 2002, 111-115.

[21] M. Hall, Jr., Solution of the Burnside problem of exponent 6. Proc. Nat. Acad. Sci. U.S.A. 43 (1957), 751-753. Zbl 0079.03003 MR 0089847

[22] F. Levi and B. van der Waerden, Über eine besondere Klasse von Gruppen. Abh. Math. Sem. Univ. Hamburg 9 (1933), no. 1, 154-158. Zbl 0005.38507 MR 3069591

[23] G. Levitt, Counting growth types of automorphisms of free groups. Geom. Funct. Anal. 19 (2009), no. 4, 1119-1146. Zbl 1196.20038 MR 2570318

[24] B. Mossé, Puissances de mots et reconnaissabilité des points fixes d'une substitution. Theoret. Comput. Sci. 99 (1992), no. 2, 327-334. Zbl 0763.68049 MR 1168468

[25] P. S. Novikov and S. I. Adjan, Infinite periodic groups. I. Izv. Akad. Nauk SSSR Ser. Mat. 32 (1968), 212-244. Zbl 0194.03301 MR 0240178

[26] P. S. Novikov and S. I. Adjan, Infinite periodic groups. II. Izv. Akad. Nauk SSSR Ser. Mat. 32 (1968), 251-524. Zbl 0194.03301 MR 0240179

[27] P. S. Novikov and S. I. Adjan, Infinite periodic groups. III. Izv. Akad. Nauk SSSR Ser. Mat. 32 (1968), 709-731. Zbl 0194.03301 MR 0240180

[28] A. Y. Ol'shanskiü, The Novikov-Adyan theorem. Mat. Sb. (N.S.) 118 (160) (1982), no. 2, 203-235, 287. Zbl 0539.20020 MR 0658789

[29] A. Y. Ol'shanskiı̌, Periodic quotient groups of hyperbolic groups. Mat. Sb. 182 (1991), no. 4, 543-567. Zbl 0820.20044 MR 1119008

[30] J.-P. Otal, Le théorème d'hyperbolisation pour les variétés fibrées de dimension 3. Astérisque 235 (1996). Zbl 0855.57003 MR 1402300 
Vol. 88 (2013)

[31] I. N. Sanov, Solution of Burnside's problem for exponent 4. Leningrad State Univ. Annals [Uchenye Zapiski] Math. Ser. 10 (1940), 166-170. Zbl 0061.02506 MR 0003397

[32] J.-P. Serre, Arbres, amalgames, $\mathrm{SL}_{2}$. Avec un sommaire anglais, Rédigé avec la collaboration de Hyman Bass, Astérisque 46, Soc. Math. France, Paris 1977. Zbl 0369.20013 MR 0476875

[33] V. A. Tartakovskiur, Solution of the word problem for groups with a $k$-reduced basis for $k>$ 6. Izvestiya Akad. Nauk SSSR. Ser. Mat. 13 (1949), 483-494. MR 0033816

Received August 26, 2010

Rémi Coulon, Department of Mathematics, Vanderbilt University, Stevenson Center 1326, Nashville, TN 37240 U.S.A.

E-mail: remi.coulon@vanderbilt.edu 